\title{
Qualidade física de Latossolo Amarelo de tabuleiros costeiros em diferentes sistemas de manejo da cana-de-açúcar
}

\author{
Romero F. B. de Vasconcelos ${ }^{1}$, Edivan R. de Souza ${ }^{2}$, José R. B. Cantalice ${ }^{3} \&$ Laércio S. Silva $^{4}$ \\ ${ }^{1}$ DTR/UFRPE. Recife, PE. E-mail: romerofalcao@hotmail.com \\ ${ }^{2}$ DEPA/UFRPE. Recife, PE. E-mail: edivanrs@hotmail.com (Autor correspondente) \\ ${ }^{3}$ DEPA/UFRPE. Recife, PE. E-mail: cantalic@terra.com.br \\ ${ }^{4}$ DEPA/UFRPE. Recife, PE. E-mail: laerciosantos18@gmail.com
}

\section{Palavras-chave:}

compactação do solo

matéria orgânica

estabilidade de agregados

\begin{abstract}
R E S U M O
Os atributos físicos do solo são indicativos imprescindíveis quando se pretende avaliar sistemas de manejo. Objetivouse, nesta pesquisa, estudar as alterações nas propriedades físicas de um Latossolo Amarelo Distrocoeso dos tabuleiros costeiros de Alagoas cultivado com cana-de-açúcar. Foram selecionadas áreas em talhões de produção de cana-deaçúcar. Os tratamentos avaliados corresponderam a área cultivada sob sistema de manejo irrigado (SMI), área sob sistema de manejo fertirrigado com vinhaça (SMFV) e área com aplicação de vinhaça + torta de filtro (SMVT), além de área de mata nativa $(\mathrm{MN})$ com fragmento de mata atlântica, tomada como referência. Avaliaram-se a densidade do solo, porosidade total do solo, diâmetro médio ponderado dos agregados, condutividade hidráulica, carbono orgânico total, grau de compactação nas profundidades de 0-0,20; 0,20-0,40 e 0,40-0,60 m. Os sistemas de manejo cultivados com cana-de-açúcar que receberam adição de resíduos da cana (SMFV + SMVT) apresentaram alterações nas propriedades físicas do solo mais favoráveis ao adequado desenvolvimento desta cultura. Dentre os diferentes sistemas de manejo cultivados com cana-de-açúcar, o que sofreu menor degradação física foi a área sob SMVT, seguido da área sob SMFV.
\end{abstract}

Key words: soil compaction organic matter aggregate stability

\section{Physical quality of Yellow Oxisol of a coastal plain under different management systems in sugarcane}

\begin{abstract}
A B S T R A C T
The physical attributes are important indicators to evaluate management systems. The objective of this research was to study alterations in the soil properties of the distrophic cohesive yellow Oxisol of a coastal plain in Alagoas state, Brazil, cultivated with sugarcane. Plots were selected in the following sugarcane-growing areas: area under irrigation management system (IMS), area fertigated with vinasse (FV), and area under application of vinasse + filter cake $(\mathrm{V}+\mathrm{FC})$. These management systems were compared to each other and to a control (native forest). The bulk soil density, total porosity, weighted mean diameter of the aggregates, hydraulic conductivity, total organic carbon and compaction degree were evaluated in 0-0.20; 0.20-0.40 e 0.40-0.60 m depths. The results showed that management system that receives residues of the sugarcane presents alterations in the physical properties of soil more adequate for development of sugarcane. Among the evaluated systems, little physical degradation was observed in area with application of vinasse + filter cake followed by area with application of vinasse.
\end{abstract}

\section{INTRODUÇÃo}

Pesquisas voltadas para a avaliação da qualidade física dos solos têm-se tornado constantes na literatura mundial em virtude das interferências na produtividade das culturas e sustentabilidade dos ecossistemas ligados ao solo. Há, também, a necessidade de se encontrar um índice de qualidade do solo, o que requer uma integração de pesquisas que abordem os atributos físicos, químicos e biológicos (Guedes et al., 2012; Yao et al., 2013; Lima et al., 2013).

A incorporação de matéria orgânica ao solo adicionada sob a forma de resíduos orgânicos melhora as propriedades físicas e químicas do solo especialmente para aqueles solos situados nos Tabuleiros Costeiros, como as classes dos Argissolos e
Latossolos. De acordo com Corrêa et al. (2008) e Vasconcelos et al. (2010) os solos dos tabuleiros costeiros cultivados com cana-de-açúcar durante longo período de tempo com sistemas de manejo com diferentes aportes de matéria orgânica, podem apresentar alterações nas propriedades físicas e químicas.

Os sistemas de manejo em cultivo tradicional promovem efeitos nas propriedades físicas verificando-se aumento da densidade do solo e diminuição da porosidade, infiltração e da condutividade hidráulica, quando comparados com o solo sob mata nativa. Oliveira et al. (2010a) constataram, investigando três sistemas de manejo da cana-de-açúcar: sequeiro, irrigado e fertirrigado, que a área com fertirrigação com vinhaça degradou menos fisicamente o solo pelo fato de lhe adicionar matéria orgânica. 
A avaliação da degradação do solo pelo fenômeno da compactação tem sido realizada com base na investigação de alguns parâmetros físicos, como densidade do solo, porosidade e condutividade hidráulica (Mcqueen \& Shepherd, 2002). A condutividade hidráulica do solo saturado é considerada um parâmetro importante na diferenciação dos efeitos dos sistemas de manejo como armazenamento de água, escoamento superficial e processos de erosão, tornando-se essencial para estudos de modelagem de processos hidrológicos (Bagarello et al., 2012, Ghanbarian-Alavijeh \& Hunt, 2012; Zimmermann et al., 2013).

De acordo com Batey \& Mckenzie (2006), existe uma variedade de critérios diagnósticos para detectar a compactação no campo. A avaliação de mudanças das propriedades físicas do solo ocasionadas pela compressão inclui a tensão do solo, a resistência à penetração, o grau de agregação, a porosidade interagregados e a densidade relativa.

O grau de compactação ou compactação relativa é a razão entre a densidade do solo e a densidade máxima do solo obtida no ensaio de Proctor (Oliveira et al., 2010b). Este parâmetro representa o grau de consolidação dos constituintes do solo (Craig, 2007) e tem sido utilizado como indicador de susceptibilidade à compactação. Carter (1990) verificou, investigando o efeito do grau de compactação ou densidade relativa do solo sobre o rendimento agrícola de cereais, o máximo de rendimento agrícola quando o grau de compactação se manteve entre 0,77 e 0,84 .

A matéria orgânica do solo repercute no valor da densidade do solo tanto pela melhoria na estabilidade estrutural do solo como pela densidade da mesma ser menor do que a dos sólidos do solo. Krzic et al. (2004) comprovaram, investigando o efeito da matéria orgânica do solo sobre o grau de compactação ou densidade relativa do solo, que o incremento de $1 \%$ no teor de carbono orgânico reduz, independentemente da textura do solo, a densidade relativa máxima do solo em 11\%; esses resultados confirmam a importância da adoção de sistemas de manejo que promovam o aumento no teor de matéria orgânica do solo.

Desta forma, o objetivo do trabalho foi investigar o efeito dos sistemas de manejo i) irrigado, ii) fertirrigado com vinhaça e iii) com aplicação de vinhaça + torta de filtro na cultura da cana-de-açúcar sobre o carbono orgânico total, densidade do solo, porosidade total, diâmetro médio ponderado de agregados, condutividade hidráulica do solo saturado e grau de compactação de um Latossolo Amarelo Distrocoeso dos tabuleiros costeiros de Alagoas. Esses sistemas de manejo foram comparados entre si e em relação a uma testemunha padrão, mata nativa $(\mathrm{MN})$ representada por solo em condições naturais de um fragmento de Mata Atlântica.

\section{MATERIAl E MÉtodos}

O experimento foi realizado na Fazenda Pau Amarelo, de propriedade da Usina Santa Clotilde S/A, localizada na latitude 9० 27' 8" Sul e Longitude 35 51' 12" Oeste, município de Rio Largo, litoral do Estado de Alagoas. O clima do local é tropical chuvoso com verão seco (As), segundo a classificação de Köppen. A precipitação média anual é de $1.752 \mathrm{~mm}$ e a temperatura média anual de $29^{\circ} \mathrm{C}$.

O solo estudado foi classificado como Latossolo Amarelo Distrocoeso (EMBRAPA, 2013), relevo plano, textura média/ argilosa. O solo é derivado do grupo Barreiras, o qual é formado por sedimentos arenoargiloso com a fração areia constituída basicamente de quartzo e a fração argila de caulinita, com baixos teores de óxidos de ferro.

Foram selecionadas áreas em talhões $(100 \times 40 \mathrm{~m}) \mathrm{de}$ produção com cana-de-açúcar representativas de três sistemas de manejo adotados pela unidade sucroalcooleira. Desta forma, os tratamentos avaliados foram: uma área cultivada em sistema de manejo irrigado (SMI), uma área cultivada em sistema de manejo de fertirrigação com vinhaça (SMFV) e uma área cultivada com a aplicação de vinhaça + torta de filtro (SMVT) e uma área testemunha, representada por solo em condições naturais de um fragmento de Mata Atlântica, denominada mata nativa $(\mathrm{MN})$. As três áreas cultivadas são exploradas com cana-de-açúcar há mais de 20 anos. Os três sistemas de manejo investigados foram adotados na unidade sucroalcooleira desde a safra 2003/2004.

Durante o período do trabalho experimental foi executado um controle rígido de tráfego de máquinas nas três áreas cultivadas com cana-de-açúcar. O preparo periódico do solo foi realizado utilizando-se subsoladores, seguido de grade de disco de dupla ação cujos órgãos ativos têm trabalhado em profundidade média de $15 \mathrm{~cm}$. Referido procedimento foi realizado utilizando-se os mesmos tratores, com frequência de duas vezes; o plantio foi manual sendo colocadas, no fundo do sulco, as canas-sementes, posteriormente cobertas com solo. Nas três áreas cultivadas a lavoura da cana-de-açúcar foi queimada antes da operação de colheita; na colheita, a cana foi cortada manualmente e transportada para o caminhão, por meio de carregadoras convencionais.

$\mathrm{Na}$ área cultivada em sistema de irrigação (SMI) foi aplicada uma lâmina de $120 \mathrm{~mm}$ de água por ciclo da cultura. $\mathrm{Na}$ adubação da cana-planta foram aplicados 40,120 e $90 \mathrm{~kg} \mathrm{ha}^{-1}$ de N, $\mathrm{P}_{2} \mathrm{O}_{5}$ e $\mathrm{K}_{2} \mathrm{O}$, respectivamente; na cana-soca foram aplicados 80,50 e $90 \mathrm{~kg} \mathrm{ha}^{-1}$ de N, $\mathrm{P}_{2} \mathrm{O}_{5}$ e $\mathrm{K}_{2} \mathrm{O}$, respectivamente.

$\mathrm{Na}$ área cultivada sob SMFV foram aplicados, por ciclo da cana-de-açúcar, $300 \mathrm{~m}^{3} \mathrm{ha}^{-1}$ de vinhaça diluída, por aspersão enquanto na adubação da cana-planta foram aplicados $40 \mathrm{~kg}$ ha ${ }^{-1}$ de $\mathrm{N}$ e na cana-soca $80 \mathrm{~kg} \mathrm{ha}^{-1} \mathrm{de} \mathrm{N}$.

$\mathrm{Na}$ área cultivada com SMVT foram aplicados $30 \mathrm{Mg} \mathrm{ha}^{-1} \mathrm{de}$ torta de filtro (Tabela 1) associada com vinhaça (Tabela 2), sendo a aplicação da mistura vinhaça + torta realizada por aspersão; já na adubação da cana-planta foram aplicados $40 \mathrm{~kg} \mathrm{ha}^{-1} \mathrm{de} \mathrm{N}$ e, na cana-soca, $80 \mathrm{~kg} \mathrm{ha}^{-1} \mathrm{de} \mathrm{N}$.

Em cada sistema de manejo foram selecionadas, ao acaso, quatro subáreas $(20 \times 5 \mathrm{~m})$ para que fossem coletadas amostras

Tabela 1. Composição química média da vinhaça diluída aplicada

\begin{tabular}{cccccccc}
\hline $\mathbf{C E}$ & \multirow{2}{*}{$\mathbf{p H}$} & $\mathbf{C}$ & $\mathbf{N}$ & $\mathbf{P}_{2} \mathbf{O}_{5}$ & $\mathbf{K}_{2} \mathbf{O}$ & $\mathbf{C a}^{2+}$ & $\mathbf{M g}^{2+}$ \\
\cline { 3 - 8 } $\mathbf{d S} \mathbf{~ m}^{-1}$ & & \multicolumn{6}{c}{$\mathbf{g ~ L}^{-1}$} \\
3,1 & 5,90 & 8,90 & 0,53 & 0,17 & 0,92 & 0,26 & 0,12 \\
\hline
\end{tabular}

CE - Condutividade elétrica 
Tabela 2. Composição química média da torta de filtro aplicada

\begin{tabular}{cccccc}
\hline $\mathbf{H}_{2} \mathbf{O}$ & $\mathbf{N}$ & $\mathbf{P}_{2} \mathbf{O}_{5}$ & $\mathbf{K}_{2} \mathbf{O}$ & $\mathbf{C a}^{2+}$ & $\mathbf{M g}^{2+}$ \\
\cline { 2 - 6 } $\mathbf{k g ~ k g}^{-1}$ & & & $\mathbf{g ~ k g}^{-1}$ & & \\
0,71 & 3,2 & 1,4 & 2,1 & 14,6 & 3,3 \\
\hline
\end{tabular}

deformadas e indeformadas de solos nas profundidades de $0-0,20 ; 0,20-0,40$ e $0,40-0,60 \mathrm{~m}$, retiradas num ponto situado nas entrelinhas de plantio da cana-de-açúcar. A coleta do solo foi realizada no mês de fevereiro de 2007.

Essas profundidades caracterizam um experimento fatorial $4 \times 3$, referente às 4 áreas avaliadas (sistemas de manejo) em 3 profundidades.

Foram determinadas a densidade do solo (Ds) pelo método do anel volumétrico, a densidade de partículas (Dp) pelo método do balão volumétrico e a porosidade total do solo de acordo com a equação: $\mathrm{P}=(1-\mathrm{Ds} / \mathrm{Dp})$. Avaliaram-se, também, o teor de carbono orgânico total e a condutividade hidráulica do solo saturado pelos métodos da EMBRAPA (1997)

A estabilidade dos agregados foi determinada via úmida adotando-se o procedimento descrito por Kemper \& Rosenau (1986), através de peneiramento em água, após pré-umedecimento lento dos agregados, por capilaridade. Os agregados assim preparados foram transferidos para dois conjuntos de cinco peneiras de 3,$35 ; 2,00 ; 1,00 ; 0,50$ e $0,25 \mathrm{~mm}$ de diâmetro de malha, sendo submetidos à agitação vertical (42 ciclos por min) durante $15 \mathrm{~min}$, imersos em recipiente com água. O solo retido em cada peneira foi acondicionado em recipientes de alumínio para secagem em estufa a $105^{\circ} \mathrm{C}$, durante $24 \mathrm{~h}$; em seguida, foram pesadas e calculas a massa e a porcentagem de agregados estáveis em cada uma das classes de diâmetro de agregados. Os valores de diâmetro médio ponderado (DMP) foram obtidos de acordo com a Eq. 1

$$
\mathrm{DMP}=\sum_{\mathrm{i}=1}^{\mathrm{n}}\left(\mathrm{X}_{\mathrm{i}} \cdot \mathrm{W}_{\mathrm{i}}\right)
$$

em que:

$\mathrm{Xi}$ - diâmetro médio das classes, $\mathrm{mm}$

Wi - proporção de cada classe em relação ao total

O grau de compactação (GC) foi definido pela relação entre a densidade do solo (Ds) e a densidade seca máxima de compactação (Dsmax), obtida pelo ensaio normal de Proctor (Oliveira et al., 2010b).

Adotou-se o delineamento em blocos ao acaso com quatro repetições (representadas pela subáreas). Os dados obtidos foram submetidos à análise de variância e as médias comparadas pelo teste de Tukey $(\mathrm{p}<0,05)$. Além disto, foram realizadas análises de correlação. A ANOVA e a comparação de médias foram realizadas de modo independente para cada profundidade estudada.

\section{Resultados e Discussão}

Verificam-se aumentos significativos nos valores de densidade do solo para os três manejos avaliados em comparação com o solo em mata nativa (Tabela 3); o mesmo comportamento foi observado para o carbono orgânico total (Tabela 3), em que a área de mata se mostrou significativamente superior à dos demais manejos.

Tabela 3. Densidade do solo (Ds) e carbono orgânico total (COT) em função dos sistemas de manejo e profundidades

\begin{tabular}{|c|c|c|c|c|}
\hline \multirow{2}{*}{$\begin{array}{c}\text { Camadas } \\
(\mathrm{m})\end{array}$} & \multicolumn{4}{|c|}{ Sistemas de manejo } \\
\hline & MN & SMI & SMVT & SMV \\
\hline & \multicolumn{4}{|c|}{ Ds $\left(\mathrm{Mg} \mathrm{m}^{-3}\right)$} \\
\hline $0-0,20$ & $1,05 \mathrm{c}$ & $1,65 a b$ & $1,49 b$ & $1,56 a b$ \\
\hline $0,20-0,40$ & $1,21 \mathrm{cb}$ & $1,72 a b$ & $1,56 \mathrm{~b}$ & $1,63 a b$ \\
\hline $0,40-0,60$ & $1,27 b$ & $1,63 a b$ & $1,58 a b$ & $1,62 a b$ \\
\hline & \multicolumn{4}{|c|}{ Carbono orgânico total $\left(\right.$ dag kg $\left.^{-1}\right)$} \\
\hline $0-0,20$ & $3,05 \mathrm{a}$ & $1,26 \mathrm{c}$ & $1,59 \mathrm{~b}$ & $1,51 \mathrm{~b}$ \\
\hline $0,20-0,40$ & $1,85 \mathrm{a}$ & $0,87 \mathrm{~b}$ & $0,85 b$ & $0,68 b$ \\
\hline $0,40-0,60$ & $1,23 \mathrm{a}$ & $0,80 \mathrm{~b}$ & $0,69 \mathrm{~b}$ & $0,60 \mathrm{~b}$ \\
\hline
\end{tabular}

Letras minúsculas iguais na mesma linha não diferem significativamente pelo teste Tukey $(\mathrm{P}$ $<0,05)$; MN - Mata nativa; SMI - Área irrigada; SMVT - Área de vinhaça + torta; SMV - Área de vinhaça

Em comparação com mata nativa na profundidade de $0-0,20$ $\mathrm{m}$, o solo em sistema de manejo irrigado apresentou maior incremento de densidade do solo (57\%), em resposta às pressões externas de compactação provocadas pelo tráfego de máquinas há mais de vinte anos. Já o menor aumento (42\%) foi observado para o sistema de manejo com aplicação de vinhaça + torta de filtro (Tabela 3). De maneira geral, o comportamento mecânico do solo em resposta às cargas externas é mais favorável para os sistemas de manejo que proporcionaram aporte no teor de matéria orgânica do solo.

O processo de compactação pelas máquinas observado nos sistemas de manejo das áreas cultivadas com cana-de-açúcar diminui o volume de poros do solo e, consequentemente, o peso por unidade de volume aumenta, sendo um indicativo de compactação do solo quando a relação entre o volume total de poros e o volume total do solo é inadequada para o máximo desenvolvimento do vegetal. O cultivo intensivo do solo tende a aumentar a densidade das camadas superficiais.

Nas profundidades de 0,20-0,40 e 0,40-0,60 m, a tendência observada para os valores de densidade do solo foi semelhante à do comportamento já descrito; todavia, o efeito dos sistemas de manejo sobre a densidade do solo foi mais evidente na camada superficial; para as camadas subsuperficiais, a ação de cargas externas de compressão é somada ao caráter coeso, característica edáfica deste solo. Comportamento semelhante para densidade do solo em sistemas de manejo com cana-deaçúcar foi observado por Oliveira et al. (2010a).

Os maiores incrementos de carbono orgânico observados na camada de 0,0-0,20 m para os manejos com adição de vinhaça e vinhaça + torta de filtro confirmam o poder de aporte de matéria orgânica no solo pelos resíduos da indústria canavieira. A matéria orgânica melhora as condições físicas do solo uma vez que é o principal agente cimentante para a formação de agregados influenciando na retenção de água, estabilidade de agregados, aeração do solo e, em contrapartida, na movimentação de água no solo. 
Observa-se uma correlação significativa $(\mathrm{p}<0,05)$ entre a Ds e o DMPu $(r=-0,8161)$ comprovando que agregados maiores resultam em menores valores para densidade do solo demonstrando que a adoção de sistemas de manejo com adição de resíduos orgânicos é uma prática benéfica para o solo (Figura 1). Vasconcelos et al. (2010), concluíram que a adição de resíduos da cana-de-açúcar exerceu ação no aumento do diâmetro médio ponderado dos agregados do solo na mesma área de estudo dessa pesquisa.

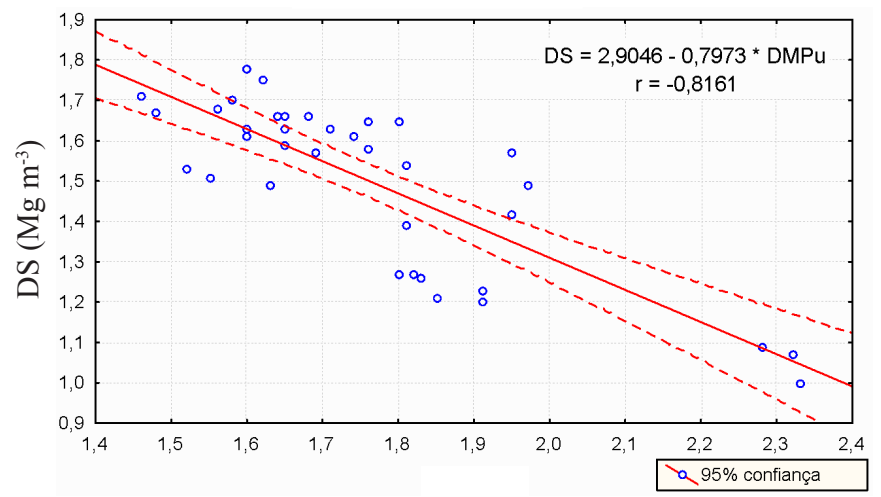

$\mathrm{DMPu}(\mathrm{mm})$

Figura 1. Relação entre a densidade do solo (Ds) e o diâmetro médio ponderado dos agregados via úmida (DMPu)

A porosidade total do solo (Tabela 4), nos diferentes sistemas de manejo, apresentou variação de 0,38 a $0,60 \mathrm{~m}^{3}$ $\mathrm{m}^{-3}$. Houve efeito significativo para o sistema de manejo. O sistema de manejo sob solo irrigado na profundidade de $0-0,20 \mathrm{~m}$, apresentou os menores valores de porosidade total do solo para todas as profundidades avaliadas, não diferindo estatisticamente do manejo com aplicação de vinhaça. O manejo que adotou a aplicação de vinhaça + torta de filtro apresentou os melhores resultados quando comparado com a área de referência. É importante frisar que essas modificações na porosidade do solo são perceptíveis até a profundidade de $60 \mathrm{~cm}$, o que faz crer que os resíduos aplicados com o passar do tempo estão sendo incorporados ao solo e melhorando as condições físicas verticalmente. Este resultado é de grande relevância, especialmente quando se trata de solos localizados nos tabuleiros costeiros e que apresentam caráter coeso em subsuperfície (Corrêa et al., 2008).

Os valores de condutividade hidráulica foram reduzidos nos solos das áreas cultivadas com cana-de-açúcar em comparação ao solo em mata nativa, nas três profundidades estudadas (Figura 2). Na profundidade de $0-0,20 \mathrm{~m}$ a maior redução

Tabela 4. Porosidade total $\left(\mathrm{m}^{3} \mathrm{~m}^{-3}\right)$ em função dos sistemas de manejo e profundidades

\begin{tabular}{ccccc}
\hline Camadas & \multicolumn{4}{c}{ Sistemas de manejo } \\
\cline { 2 - 5 }$(\mathbf{m})$ & SMI & MN & SMVT & SMV \\
$0-0,20$ & $0,38 \mathrm{c}$ & $0,60 \mathrm{a}$ & $0,43 \mathrm{~b}$ & $0,41 \mathrm{c}$ \\
$0,20-0,40$ & $0,35 \mathrm{c}$ & $0,54 \mathrm{a}$ & $0,42 \mathrm{~b}$ & $0,38 \mathrm{c}$ \\
$0,40-0,60$ & $0,39 \mathrm{c}$ & $0,53 \mathrm{a}$ & $0,41 \mathrm{~b}$ & $0,39 \mathrm{c}$ \\
\hline
\end{tabular}

Letras minúsculas iguais na mesma linha não diferem significativamente pelo teste Tukey $(\mathrm{P}$ $<0,05)$; MN - Mata nativa; SMI - Área irrigada; SMVT - Área de vinhaça + torta; SMV - Área de vinhaça
A.

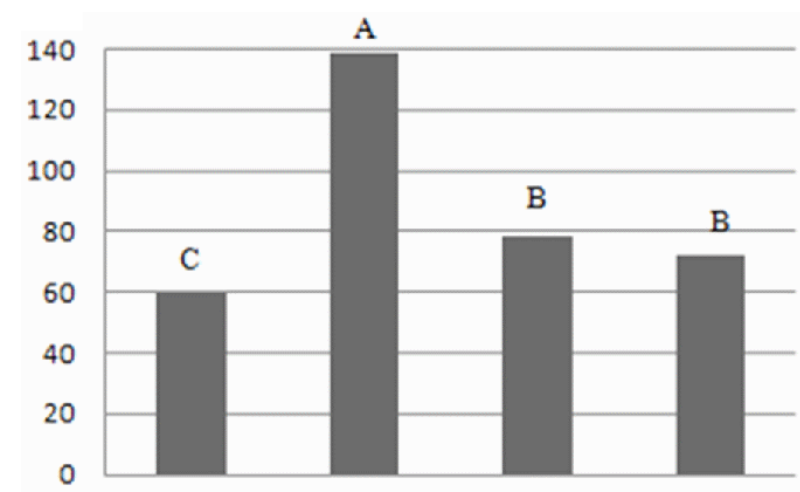

B.

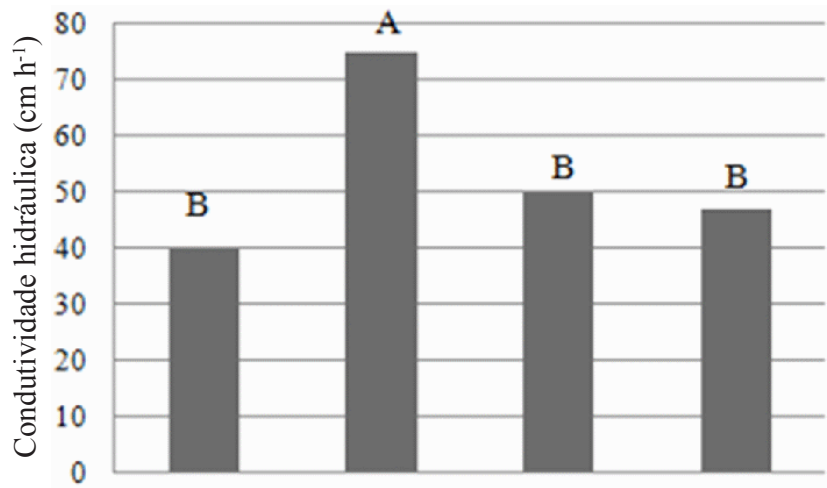

C.

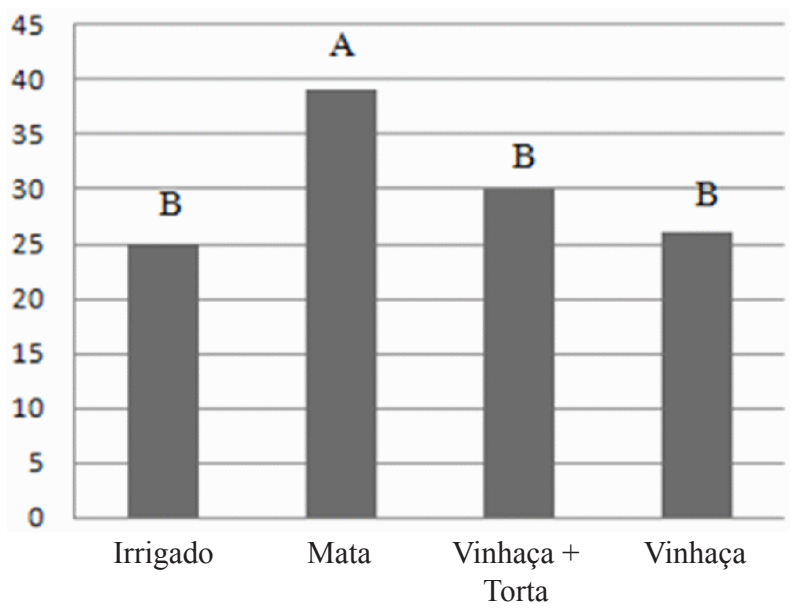

Figura 2. Condutividade hidráulica saturada em função dos sistemas de manejo: mata nativa, área irrigada, área de vinhaça + torta de filtro e área de vinhaça nas profundidades 0-0,20 (A), 0,20-0,40 (B) e $0,40-0,60 \mathrm{~m}(\mathrm{C})$

(57\%) foi constatada na área com irrigação. A condutividade hidráulica saturada sofreu redução em resposta à compactação do solo, provocada pelo cultivo intensivo. Este comportamento pode estar relacionado à reorganização do sistema poroso, ou seja, da distribuição do diâmetro e não continuidade vertical dos poros (Marsili et al., 1998).

Valores semelhantes foram encontrados por Marques et al. (2008), avaliando a condutividade hidráulica saturada em Latossolo Amarelo Distrófico no Baixo Amazonas. Esses autores obtiveram valores de $\mathrm{K}_{0}$ de $102,75 \mathrm{~cm} \mathrm{~h}^{-1}$ no horizonte superficial $(0-16 \mathrm{~cm})$ e de $22,50 \mathrm{~cm} \mathrm{~h}^{-1}$ no horizonte Bw1 $(46-65 \mathrm{~cm})$. 
Já Barbosa et al. (2008) observaram, avaliando o efeito da aplicação de lodo de esgoto e consequente incremento de matéria orgânica num Latossolo Vermelho eutroférrico valores de $\mathrm{K}_{0}$ para o tratamento testemunha $\left(0 \mathrm{Mg} \mathrm{ha}^{-1}\right)$ e $12 \mathrm{Mg} \mathrm{ha}^{-1}$ de 6,05 e $43,10 \mathrm{~cm} \mathrm{~h}^{-1}$, respectivamente.

A redução da condutividade hidráulica saturada é imputada também ao decréscimo da macroporosidade com o aumento da densidade do solo, uma vez que o movimento de água no solo está diretamente associado à porosidade de drenagem. Solos com baixa condutividade hidráulica são mais susceptíveis à erosão devido à maior quantidade de água que permanece na superfície resultando em maior escoamento superficial e, consequentemente, maiores perdas de solo e água. Portanto, os solos das áreas cultivadas com cana-de-açúcar apresentam maior risco de degradação e, desses, o solo em sistema de irrigação se encontra em posição mais desfavorável.

Observa-se aumento do grau de compactação dos solos com sistemas de manejo com cana-de-açúcar, em comparação com o solo em mata nativa (Tabela 5).

Tabela 5. Grau de compactação do solo (GC) em função dos sistemas de manejo e profundidades

\begin{tabular}{ccccc}
\hline Camada & \multicolumn{4}{c}{ Sistemas de manejo } \\
\cline { 2 - 5 }$(\mathbf{m})$ & SMI & MN & SMVT & SMV \\
$0-0,20$ & $0,91 \mathrm{a}$ & $0,74 \mathrm{~b}$ & $0,88 \mathrm{a}$ & $0,90 \mathrm{a}$ \\
$0,20-0,40$ & $0,99 \mathrm{a}$ & $0,82 \mathrm{~b}$ & $0,94 \mathrm{a}$ & $0,98 \mathrm{a}$ \\
$0,40-0,60$ & $0,94 \mathrm{a}$ & $0,86 \mathrm{~b}$ & $0,94 \mathrm{a}$ & $0,96 \mathrm{a}$ \\
\hline
\end{tabular}

Letras minúsculas iguais na mesma linha não diferem significativamente pelo teste Tukey $(P$ $<0,05)$. MN - Mata nativa; SMI - Área irrigada; SMVT - Área de vinhaça + torta; SMV - Área de vinhaça

Constata-se também um aumento nos valores do grau de compactação com as profundidades estudadas. Conforme Carter (1990), quando o grau de compactação (GC) supera a faixa de 0,86 a 0,90 ocorre uma grande redução nos macroporos afetando os movimentos dos fluidos e diminuindo a capacidade de desenvolvimento dos vegetais. Tavares et al. (2012) obtiveram, avaliando um Argissolo Coeso em Pernambuco cultivado com cana de açúcar valores de grau de compactação variando de 0,76 a 0,89 .

Para a profundidade $0-0,20 \mathrm{~m}$ apenas os solos em mata nativa $(\mathrm{GC}=0,74)$ e vinhaça + torta de filtro $(\mathrm{GC}=0,88)$ ficaram abaixo do limite crítico para o grau de compactação proposto por Carter (1990). O grau de compactação não é suficiente para caracterizar o processo de compactação do solo sendo considerado apenas um indicador de prováveis limitações ao desenvolvimento das plantas (Betioli Júnior et al., 2012).

Os resultados do grau de compactação para as áreas em cultivo com cana-de-açúcar denotam que o aporte de matéria orgânica proporcionado pelos sistemas de manejo ainda não foi suficiente para provocar as alterações esperadas; melhores resultados não foram alcançados devido, possivelmente, à queima da cana-de-açúcar antes da colheita. Esta prática cultural contribui para a perda do carbono orgânico do solo. Suzuki et al. (2007) enfatizam que a relação do grau de compactação com as propriedades físicas do solo e culturas ainda deve ser testada para que sejam definidos os valores críticos de grau de compactação encontrados no desenvolvimento das plantas.
Constatou-se que o grau de compactação apresentou correlação significativa $(\mathrm{p}<0,01)$ e negativa $(\mathrm{r}=-0,7797)$ com o diâmetro médio ponderado dos agregados via úmida $(\mathrm{DMPu})$, evidenciando uma boa relação funcional entre as duas variáveis para o solo estudado (Figura 3). A relação funcional demonstra que o aumento do diâmetro médio ponderado úmido reduz o grau de compactação indicando a manutenção de sistemas de manejo que forneçam matéria orgânica ao solo e a adoção de novas práticas culturais na lavoura da cana-de-açúcar.

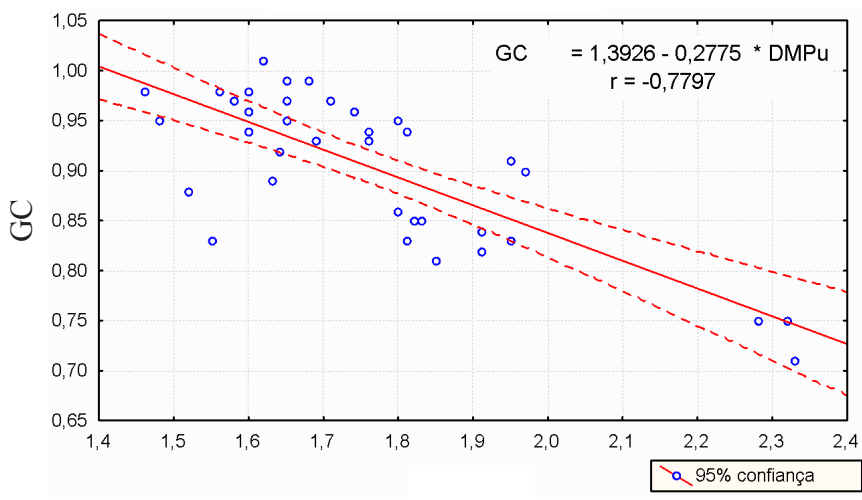

$\mathrm{DMPu}(\mathrm{mm})$

Figura 3. Relação entre o grau de compactação (GC) e o diâmetro médio ponderado dos agregados via úmida (DMPu)

\section{Conclusões}

1. Os sistemas de manejo do solo cultivado com cana-deaçúcar provocaram alterações nos atributos físicos do solo aumentando a densidade e o grau de compactação e reduzindo a condutividade hidráulica saturada quando comparados com a mata nativa.

2. Os sistemas de manejo com aportes de resíduos (SMFV e SMVT) da cana-de-açúcar apresentaram menor degradação física do solo.

\section{Literatura Citada}

Bagarello, V.; D'Asaro, F.; Iovino, M. field assessment of the simplified falling head technique to measure the saturated soil hydraulic conductivity. Geoderma, v.187-188 p.49-58, 2012.

Barbosa, G. M. C.; Tavares Filho, J.; Fonseca, I. C. B. Condutividade hidráulica saturada e não saturada de Latossolo Vermelho Eutroférrico tratado com lodo de esgoto. Revista Brasileira de Ciência do Solo, v.28, p.403407, 2008.

Batey, T.; Mckenzie, D. C. Soil compaction: Identification directly in the field. Soil Use and Management, v.22, p.123131, 2006.

Betioli Júnior, E.; Moreira, W. H.; Tormena, C. A.; Ferreira, C. J. B.; Silva, A. P.; Giarola, N. F. B. Intervalo hídrico ótimo e grau de compactação de um Latossolo vermelho após 30 anos sob plantio direto. Revista Brasileira de Ciência do Solo, v.36, p.971-982, 2012. 
Carter, M. R. Relative measures of soil bulk density to characteterize compaction in tillage studies of fine loamy sands. Canadian Journal Soil Science, v.70 p.425-433, 1990.

Corrêa, M. M.; Ker, J. C.; Barrón, V.; Torrent, J.; Curi, N.; Torres, T. C. P. Caracterização física, química, mineralógica e micromorfológica de horizontes coesos e fragipãs de solos vermelhos e amarelos do ambiente Tabuleiros Costeiros. Revista Brasileira de Ciência do Solo, v.32, p.297-313, 2008.

Craig, R. F. Mecânica dos solos. Rio de Janeiro: Livros Técnicos e Científicos, 2007.365p.

EMBRAPA - Empresa Brasileira de Pesquisa Agropecuária. Manual de métodos de análise de solo. Rio de Janeiro: EMBRAPA, 1997. 212p.

EMBRAPA - Empresa Brasileira de Pesquisa Agropecuária. Centro Nacional de Pesquisa de Solos. Sistema brasileiro de classificação de solos. 3.ed. Brasília: EMBRAPA, 2013.353p.

Ghanbarian-Alavijeh, B.; Hunt, A. G. Unsaturated hydraulic conductivity in porous media: Percolation theory. Geoderma, v.187/188, p.77-84, 2012.

Guedes, E. M. S.; Fernandes, A. R.; Lima, H. V.; Serra, A. P.; José Costa, J. R.; Guedes, R. S. Impacts of different management systems on the physical quality of an amazonian oxisol. Revista Brasileira de Ciência do Solo, v.36, p.1269-1277, 2012.

Kemper, W. D.; Rosenau, R. C. Aggregate stability and size distribution. In: Klute, A. Methods of soil analysis. Madison: American Society of Agronomy, 1986. Part 1, p.425-442. Agronomy, 9

Krzic, M.; Buhner, C. E.; Teste, F.; Dompter, L.; Rahman, S. Soil properties influencing compactability of forest soils in British Columbia. Canadian Journal Soil Science, v.84, p.219-236, 2004.

Lima, A. C. R.; Brussaard, L.; Totola, M. R.; Hoogmoed, W. B.; Goede, R. G. M. de. A functional evaluation of three indicator sets for assessing soil quality. Applied Soil Ecology, v.64, p.194-200, 2013.

Marques, J. D. O.; Teixeira, W. G.; Reis, A. M.; Cruz Júnior, O, F; Martins, G. C. Avaliação da condutividade hidráulica do solo saturada utilizando dois métodos de laboratório numa topossequência com diferentes coberturas vegetais no Baixo Amazonas. Acta Amazonica, v.38, p.193-206, 2008.
Marsili, A.; Servadio, P.; Pagliai, M.; Vignozzi, N. Changes of some physical properties of a clay soil following passage of rubber-and metal-tracked tractors. Soil and Tillage Research, v.49, p.185-199, 1998.

Mcqueen, D. J.; Shepherd, T. G. Physical changes and compaction sensitivity of a fine-textured, poorly drained soil (Typic Endo-aquept) under varying durations of cropping, Manawatu Region, New Zealand. Soil and Tillage Research, v.63, p.93-107, 2002.

Oliveira, V. S.; Rolim, M. M.; Vasconcelos, R. F. B.; Costa, Y. D. J.; Pedrosa, E. M. R. Compactação de um Argissolo Amarelo Distrocoeso submetido a diferentes manejos. Revista Brasileira de Engenharia Agrícola e Ambiental, v.14, p.914-920, 2010a.

Oliveira, V. S.; Rolim, M. M.; Vasconcelos, R. F. B.; Pedrosa, E. M. R. Distribuição de agregados e carbono orgânico em um Argissolo Amarelo distrocoeso em diferentes manejos. Revista Brasileira de Engenharia Agrícola e Ambiental, v.14, p.907-913, 2010b.

Suzuki, L. E. A. S.; Reichert, J. M.; Reinert, D. J.; Lima, C. L. R. Grau de compactação, propriedades físicas e rendimento de culturas em Latossolo e Argissolo. Pesquisa Agropecuária Brasileira, v.42, p.1159-1167, 2007.

Tavares, U. E.; Rolim, M. M.; Pedrosa, E. M. R.; Montenegro, A. A. A.; Magalhães, A. G.; Barreto, M. T. L. Variabilidade espacial de atributos físicos e mecânicos de um Argissolo sob cultivo de cana-de-açúcar. Revista Brasileira de Engenharia Agrícola e Ambiental, v.16, p.1206-1214, 2012.

Vasconcelos, R. F. B. de; Cantalice, J. R. B.; Oliveira, V. S.; Costa, Y. D. J.; Cavalcante, D. M. Estabilidade de agregados de um Latossolo Amarelo Distrocoeso de Tabuleiro Costeiro sob diferentes aportes de resíduos orgânicos da cana-de-açúcar. Revista Brasileira de Ciência do Solo, v.34, p.309-316, 2010.

Yao, R.; Yang, J.; Gao, P.; Zhang, J.; Jin, W. Determining minimum data set for soil quality assessment of typical salt-affected farmland in the coastal reclamation area. Soil \& Tillage Research, v.128, p.137-148, 2013.

Zimmermann, A.; Schinn, D. S.; Francke, T.; Elsenbeer, H.; Zimmermann, B. Uncovering patterns of near-surface saturated hydraulic conductivity in an overland flowcontrolled landscape. Geoderma, v.195/196, p.1-11, 2013 